\title{
Properties of six short-period massive binaries: A study of the effects of binarity on surface chemical abundances ${ }^{\star}$
}

\author{
F. Martins ${ }^{1}$, L. Mahy ${ }^{2,3, \star \star}$, and A. Hervé ${ }^{1}$ \\ ${ }^{1}$ LUPM, Université de Montpellier, CNRS, Place Eugène Bataillon, 34095 Montpellier, France \\ e-mail: fabrice.martins@umontpellier.fr \\ 2 Space sciences, Technologies, and Astrophysics Research (STAR) Institute, Université de Liège, Quartier Agora, Bât. B5c, \\ Allée du 6 aout, 19c, 4000 Liège, Belgium \\ 3 Instituut voor Sterrenkunde, KU Leuven, Celestijnenlaan 200D, Bus 2401, 3001 Leuven, Belgium
}

Received 18 July 2017 / Accepted 1 September 2017

\begin{abstract}
Context. A significant percentage of massive stars are found in multiple systems. The effect of binarity on stellar evolution is poorly constrained. In particular, the role of tides and mass transfer on surface chemical abundances is not constrained observationally. Aims. The aim of this study is to investigate the effect of binarity on the stellar properties and surface abundances of massive binaries. Methods. We performed a spectroscopic analysis of six Galactic massive binaries. We obtained the spectra of individual components via a spectral disentangling method and subsequently analyzed these spectra by means of atmosphere models. The stellar parameters and CNO surface abundances were determined.

Results. Most of these six systems are comprised of main-sequence stars. Three systems are detached, two are in contact, and no information is available for the sixth system. For 11 out of the 12 stars studied, the surface abundances are only mildly affected by stellar evolution and mixing. The surface abundances are not different from those of single stars within the uncertainties. The secondary of XZ Cep is strongly chemically enriched. Considering previous determinations of surface abundances in massive binary systems suggests that the effect of tides on chemical mixing is limited, whereas the mass transfer and removal of outer layers of the mass donor leads to the appearance of chemically processed material at the surface, although this is not systematic. The evolutionary masses of the components of our six systems are on average $16.5 \%$ higher than the dynamical masses. Some systems seem to have reached synchronization, while others may still be in a transitory phase.
\end{abstract}

Key words. stars: massive - binaries: close - binaries: spectroscopic - stars: atmospheres - stars: abundances

\section{Introduction}

The evolution of massive stars depends on several physical processes, of which the main processes are mass loss and rotation. Mass loss removes material from the outer layers, thereby reducing the mass of the star and thus affecting its internal structure and luminosity (Chiosi \& Maeder 1986). Main-sequence massive stars rotate on average faster than any other main-sequence star. Their average projected rotational velocity is found to be between 100 and $150 \mathrm{~km} \mathrm{~s}^{-1}$ depending on metallicity (Penny \& Gies 2009; Huang et al. 2010; Ramírez-Agudelo et al. 2013). Rotation flattens the star, which triggers the transport of angular momentum and chemical species from the core to the surface (Maeder \& Meynet 2000). Rotation also modifies mass loss rates. Other processes affect the evolution of massive stars. The presence of a strong dipolar magnetic field, which is present in about 7\% of OB stars (Grunhut et al. 2017) can alter both mass loss and rotation (Ud-Doula et al. 2009; Meynet et al. 2011). The presence of a companion can also modify the evolution of a star through tides and mass transfer (e.g., Mandel \& de Mink 2016).

\footnotetext{
* Based on observations made with the SOPHIE spectrograph on the $1.93 \mathrm{~m}$ telescope at Observatoire de Haute-Provence (OHP, CNRS/AMU), France.

$\star \star$ F.R.S.-FNRS Postdoctoral researcher.
}

A number of massive OB stars are found in binary systems. However, the fraction of multiple systems is not fully established and varies depending on the samples and environment. Kobulnicky et al. (2014) reported that 35\% (55\% after corrections for biases) of the $128 \mathrm{OB}$ stars they studied in Cyg OB2 definitely have a companion. Sana et al. (2012) found a binary fraction of 56\% (69\% after corrections) for six Galactic open clusters and a total of 71 systems (40 definite binaries); Sana et al. (2014) detected a companion in 53\% of a sample of 96 Galactic O stars and claimed a binary fraction of 91\% accounting for known systems not detected in their survey (many systems having large separations though); and in the Large Magellanic Cloud (LMC), Sana et al. (2013) determined an observed fraction of 35\% (corrected to 51\%) among $360 \mathrm{O}$ stars (126 definite binaries). A minimum fraction of $21 \%$ was determined by Mahy et al. (2013) for four Cygnus OB associations (19 systems, 4 definite binaries). In addition, Kobulnicky et al. (2014) and Sana et al. (2012) found a rather uniform distribution of mass ratios and a weak decrease of the period distribution among definite binaries. The latter distribution extends up to several thousands of days.

The presence of a companion around a significant number of OB stars raises the question of the effects of this presence on stellar evolution. Interactions are expected in close systems. For wide separations, both components evolve as single stars. There are two main categories of effects caused by the 
presence of a close companion: those due to tides and those due to mass transfer. The former trigger energy exchange between the system and its components. The consequence is a change of the internal structure under the influence of energy dissipation (and thus heating) through radiative damping, viscous friction, and (gravito-)inertial waves (Zahn 1989; Mathis \& Remus 2013). The geometry of the star and its internal rotation profile can also be modified. Hence the transport of angular momentum and chemical species are affected. In practice, these effects lead to synchronization of the rotational and orbital periods (Zahn 1977). This can spin up or spin down individual components and thus amplify or reduce the effects of rotation on their evolution. Mass transfer also drastically affects the evolution of massive stars. Owing to the mass-luminosity relation, a decrease of mass implies a reduction of luminosity, and thus a change of the path in the Hertzsprung-Russell (HR) diagram (Wellstein et al. 2001). The mass donor loses its external layers, thereby revealing hotter internal regions with different chemical properties at the surface. The mass gainer is polluted by material from the donor and its envelope is mixed with new material (Langer et al. 2008). Together with mass, angular momentum is exchanged. This affects the rotational properties of both stars, in a way that depends on the efficiency of mass transfer. The outcome of mass transfer depends on the orbital properties of the system (mass ratio, separation) and on the intrinsic evolution of the components.

Stellar evolution in (massive) binaries is thus complex. Constraints from analysis of dedicated systems are therefore highly requested. One of the expectations of binary evolution is that surface abundances are modified compared to single stars. Indeed, mixing processes due to rotation depend on the internal structure of the star, which is affected by tides (de Mink et al. 2009). Contamination by accretion in mass transfer episodes obviously affects surface chemical patterns (Langer et al. 2008). In this case, the mass donor loses its external layers so that its surface is physically moved to deeper layers where chemical composition corresponds to more processed material. These effects of binarity on surface abundances are one of the possibilities quoted to explain peculiar abundance patterns. For instance, the nitrogen-rich stars with low projected rotational velocity, and their counterparts, nitrogen-poor stars with high $V \sin i$, reported by Hunter et al. (2008) - see also Grin et al. (2017) - may be the result of such interactions. However the difference between the chemical patterns of binary and single stars has not been established observationally. The attempt performed by Garland et al. (2017) on a sample of B-type binaries did not show any clear difference, but only the primary component of their systems was analyzed.

Here we present a study of six Galactic massive binaries. Our goal is to determine the stellar parameters and surface CNO abundances of their components in order to investigate whether they are different from presumably single stars. Section 2 describes our sample and the spectroscopic observations. Data analysis and the associated results are gathered in Sect. 3. Results are discussed in Sect. 4. Finally we summarize our conclusions in Sect. 5.

\section{Sample and observations}

In order to test the effects of binarity on the properties and evolution of massive stars, we selected six short period systems $(P<6$ days $)$ from the compilation of Gies (2003). All systems except DH Cep are eclipsing binaries. The targets presented in this study were observed at Observatoire de Haute-Provence with the SOPHIE instrument (Bouchy et al. 2013) during the nights of 22 to 26 August 2013. The SOPHIE instrument delivers
Table 1. Observational information.

\begin{tabular}{lcccc}
\hline \hline Star & ST & $\begin{array}{c}\text { Period } \\
{[\mathrm{d}]}\end{array}$ & $V$ & $\mathrm{Nb}$. \\
\hline DH Cep & O5.5V-III+O6V-III & 2.11095 & 8.61 & 11 \\
V382 Cyg & O6.5V((f))+O6V((f)) & 1.88555 & 8.65 & 10 \\
Y Cyg & O9V+O9.5V & 2.99633 & 7.32 & 9 \\
V478 Cyg & O9.5V+O9.5V & 2.88086 & 8.68 & 10 \\
XZ Cep & O9.5V+B1III & 5.09725 & 8.51 & 6 \\
AH Cep & B0.2V+B2V & 1.77473 & 6.88 & 15 \\
\hline
\end{tabular}

Notes. The table includes: target name, spectral type, period, $V$ magnitude, and number of spectra (Nb) obtained for each system.

high spectral resolution échelle spectra in the 3900-6900 $\AA$ wavelength range. The main properties of the observed binaries are listed in Table 1 . The high efficiency mode of SOPHIE, corresponding to a spectral resolution $R=39000$, was used. The spectra were obtained in 15 to 40 min depending on the brightness of the target and have a signal-to-noise ratio close to 200 in the continuum. The observing strategy (number of observations per system) was adjusted each night in order to ensure an optimal coverage of the phases of maximum separation between the components of the systems, as requested for a good spectral disentangling. Data reduction was performed automatically by the SOPHIE pipeline (Bouchy et al. 2009) adapted from the ESO/HARPS software.

\section{Modeling and spectroscopic analysis}

\subsection{Orbital solutions and spectral disentangling}

To compute the orbital solution of each system, we first measured the Radial Velocities (RVs) of each component. For this purpose, we fit the spectral lines of helium with the highest ionization stage. The higher the ionization stage, the closer to the photosphere the lines are formed. The computations of the RVs were performed by adopting the rest wavelength from Conti et al. (1977). We then used the Liège Orbital Solution Package (LOSP ${ }^{1}$ ) to determine the SB2 orbital solution of the system. Since the orbital periods of these systems are well known, we decided to fix these periods in the computations. Other than Y Cyg, the other systems all have a circular orbit. The orbital solutions are shown in Fig. 1. The corresponding parameters are gathered in Table 2.

We then used the orbital parameters as inputs to our spectral disentangling code. We applied the Fourier approach of Hadrava (1995) to separate the spectral contributions of each component in the different systems. This method uses the Nelder \& Mead downhill simplex method on the multidimensional parameter space to reach the best $\chi^{2}$ fit between the recombined component spectra and the observed data. Discussions about the spectral disentangling method used in this paper can be found in Pavlovski \& Hensberge (2010) and in Mahy et al. (2017). Because the eclipses are not total in the different systems, the output composite spectra must be renormalized and corrected for

1 The LOSP is developed and maintained by $\mathrm{H}$. Sana. The algorithm is based on the generalization of the SB1 method of Wolfe et al. (1967) to the SB2 case along the lines described in Rauw et al. (2000) and Sana et al. (2006). It is available at http://www.stsci.edu/ ihsana/losp. html. 

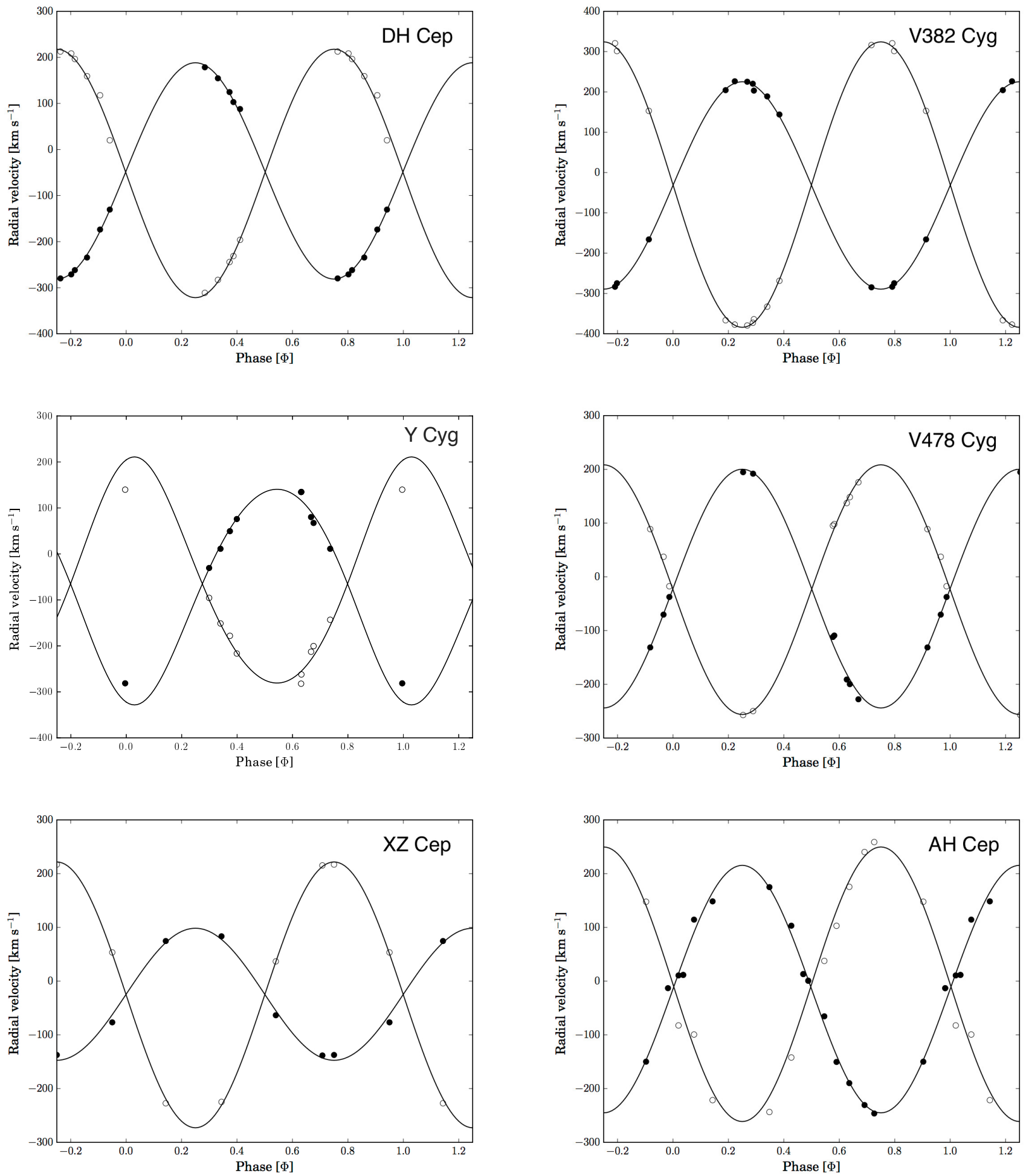

Fig. 1. Orbital solutions (solid) lines and measured RVs (circles) for the primary (filled symbols) and secondary (open circle) of each system.

the brightness ratios. We estimated a first approximation of the brightness ratio by computing the equivalent widths of several spectral lines (Si IV 4089, He I 4143, He I 4471, He II 4542, and O III 5592) and by comparing these values to those calculated from synthetic spectra corresponding to stars with the same spectral types as the components of our sample. Then, we iteratively adjusted the brightness ratios by computing such ratios from the ratios between the luminosities of the two components; i.e., luminosities that were obtained from the effective temperature and radius of each star.

\subsection{Stellar parameters}

We used the Fourier transform method (Gray 1976; SimónDíaz \& Herrero 2007) to determine the projected rotational velocity $V \sin i$. When the combined spectra showed sufficiently wide separations between both components of the system so that individual lines could be clearly isolated, we used the combined spectrum directly. Otherwise, we relied on the disentangled spectra. The uncertainty of our determinations is $10 \mathrm{~km} \mathrm{~s}^{-1}$. We found that all stars have $V \sin i$ larger than $100 \mathrm{~km} \mathrm{~s}^{-1}$. 
Table 2. Orbital solution for the sample stars.

\begin{tabular}{|c|c|c|c|c|c|c|}
\hline & \multicolumn{2}{|c|}{ AH Cep } & \multicolumn{2}{|c|}{ DH Cep } & \multicolumn{2}{|c|}{ XZ Cep } \\
\hline & Primary & Secondary & Primary & Secondary & Primary & Secondary \\
\hline$P$ [day] & \multicolumn{2}{|c|}{1.774727 (fixed) } & \multicolumn{2}{|c|}{2.11095 (fixed) } & \multicolumn{2}{|c|}{5.097253 (fixed) } \\
\hline$e^{L \ln s}$ & \multicolumn{2}{|c|}{0.0 (fixed) } & \multicolumn{2}{|c|}{0.0 (fixed) } & \multicolumn{2}{|c|}{0.0 (fixed) } \\
\hline$\omega\left[^{\circ}\right]$ & \multicolumn{2}{|c|}{-} & \multicolumn{2}{|c|}{-} & \multicolumn{2}{|c|}{-} \\
\hline$T_{0}[\mathrm{HJD}-2450000]$ & \multicolumn{2}{|c|}{$6526.783 \pm 0.008$} & \multicolumn{2}{|c|}{$6525.564 \pm 0.006$} & \multicolumn{2}{|c|}{$6522.656 \pm 0.014$} \\
\hline$q\left(M_{1} / M_{2}\right)$ & \multicolumn{2}{|c|}{$1.132 \pm 0.056$} & \multicolumn{2}{|c|}{$1.149 \pm 0.020$} & \multicolumn{2}{|c|}{$2.013 \pm 0.092$} \\
\hline$\gamma\left[\mathrm{km} \mathrm{s}^{-1}\right]$ & $-14.69 \pm 5.29$ & $-13.43 \pm 5.53$ & $-46.43 \pm 2.47$ & $-51.95 \pm 2.65$ & $-24.47 \pm 3.49$ & $-25.58 \pm 4.39$ \\
\hline$K\left[\mathrm{~km} \mathrm{~s}^{-1}\right]$ & $236.46 \pm 7.33$ & $267.57 \pm 8.29$ & $234.81 \pm 3.97$ & $269.70 \pm 4.56$ & $122.86 \pm 3.49$ & $247.29 \pm 7.03$ \\
\hline$a \sin i\left[R_{\odot}\right]$ & $8.29 \pm 0.26$ & $9.38 \pm 0.29$ & $9.79 \pm 0.17$ & $11.24 \pm 0.19$ & $12.37 \pm 0.35$ & $24.89 \pm 0.71$ \\
\hline$M \sin ^{3} i\left[M_{\odot}\right]$ & $12.49 \pm 0.78$ & $11.04 \pm 0.66$ & $15.01 \pm 0.66$ & $13.07 \pm 0.57$ & $17.88 \pm 1.14$ & $8.88 \pm 0.46$ \\
\hline \multirow[t]{3}{*}{$\mathrm{rms}\left[\mathrm{km} \mathrm{s}^{-1}\right]$} & \multicolumn{2}{|c|}{14.36} & \multicolumn{2}{|c|}{8.78} & \multicolumn{2}{|c|}{9.09} \\
\hline & \multicolumn{2}{|c|}{ V382 Cyg } & \multicolumn{2}{|c|}{ V478 Cyg } & \multicolumn{2}{|c|}{ Y Cyg } \\
\hline & Primary & Secondary & Primary & Secondary & Primary & Secondary \\
\hline$P$ [day] & \multicolumn{2}{|c|}{1.885545 (fixed) } & \multicolumn{2}{|c|}{2.880867 (fixed) } & \multicolumn{2}{|c|}{2.9963316 (fixed) } \\
\hline$e^{L \ln s}$ & \multicolumn{2}{|c|}{0.0 (fixed) } & \multicolumn{2}{|c|}{0.0 (fixed) } & \multicolumn{2}{|c|}{$0.128 \pm 0.016$} \\
\hline$\omega\left[^{\circ}\right]$ & \multicolumn{2}{|c|}{-} & \multicolumn{2}{|c|}{-} & \multicolumn{2}{|c|}{$168.63 \pm 4.95$} \\
\hline$T_{0}[\mathrm{HJD}-2450000]$ & 6527.020 & \pm 0.003 & 6525.76 & \pm 0.006 & 6526.45 & \pm 0.041 \\
\hline$q\left(M_{1} / M_{2}\right)$ & $1.376=$ & 0.009 & $1.046=$ & 0.040 & $1.053=$ & 0.017 \\
\hline$\gamma\left[\mathrm{km} \mathrm{s}^{-1}\right]$ & $-32.04 \pm 1.30$ & $-29.88 \pm 1.60$ & $-21.87 \pm 3.80$ & $-23.87 \pm 3.86$ & $-68.55 \pm 2.90$ & $-61.47 \pm 2.99$ \\
\hline$K\left[\mathrm{~km} \mathrm{~s}^{-1}\right]$ & $257.32 \pm 1.46$ & $354.11 \pm 2.01$ & $222.11 \pm 5.43$ & $232.28 \pm 5.67$ & $232.41 \pm 3.00$ & $244.81 \pm 3.16$ \\
\hline$a \sin i\left[R_{\odot}\right]$ & $9.58 \pm 0.05$ & $13.19 \pm 0.08$ & $12.64 \pm 0.31$ & $13.22 \pm 0.32$ & $13.64 \pm 0.18$ & $14.37 \pm 0.19$ \\
\hline$M \sin ^{3} i\left[M_{\odot}\right]$ & $25.85 \pm 0.38$ & $18.78 \pm 0.27$ & $14.31 \pm 0.72$ & $13.68 \pm 0.68$ & $16.87 \pm 0.53$ & $16.02 \pm 0.50$ \\
\hline $\mathrm{rms}\left[\mathrm{km} \mathrm{s}^{-1}\right]$ & 5 . & & 10 & & 12 & \\
\hline
\end{tabular}

Notes. $1 \sigma$ errors are given. For circular systems, $T_{0}$ refers to the time of the conjunction (primary in front) and for the eccentric system, $T_{0}$ refers to the periastron passage. The parameters $\gamma, K$, and $a \sin i$ denote the apparent systemic velocity, the semiamplitude of the RV curve, and the projected separation between the center of the star and the center of mass of the binary system.

To estimate the amount of extra broadening (macroturbulence), we used synthetic spectra (see below) with $T_{\text {eff }}$ and $\log g$ consistent with the spectral type and luminosity class of each star (Martins et al. 2005; Nieva \& Przybilla 2014). We convolved these spectra with a rotational profile adopting the $V \sin i$ derived from the Fourier transform method. We added an extra broadening by means of a radial-tangential profile parameterized by a velocity $v_{\mathrm{mac}}$. Comparisons with the observed, disentangled spectrum of each component revealed that for most systems there was no need for an extra broadening. We thus adopted $v_{\text {mac }}=$ $0 \mathrm{~km} \mathrm{~s}^{-1}$. We only chose $v_{\text {mac }}=100 \mathrm{~km} \mathrm{~s}^{-1}$ for DH Cep to correctly reproduce the line wings.

We determined $T_{\text {eff }}$ and $\log g$ from the classical diagnostics: the helium ionization balance for $T_{\text {eff }}$ and the Balmer lines broadening for the surface gravity (e.g., Martins 2011). For B stars we replaced helium by silicon for the effective temperature determination. We relied on a grid of synthetic spectra computed with the code CMFGEN (Hillier \& Miller 1998). NonLTE atmosphere models were computed for stars with $21000<$ $T_{\text {eff }}<45000 \mathrm{~K}$ and $3.0<\log g<4.3$. Luminosities were assigned according to the calibration of Martins et al. (2005) and mass loss rates were computed from Vink et al. (2001). Wind terminal velocities were estimated from $v_{\infty}=3.0 \times v_{\text {esc }}$ (Garcia et al. 2014). A solar composition (Grevesse et al. 2010) was assumed and the following elements were included: $\mathrm{H}$, $\mathrm{He}, \mathrm{C}, \mathrm{N}, \mathrm{O}, \mathrm{Ne}, \mathrm{Mg}, \mathrm{Si}, \mathrm{S}, \mathrm{Ar}, \mathrm{Ca}, \mathrm{Fe}$, and Ni. Once atmosphere models were converged, a formal solution of the radiative transfer equation led to the synthetic emergent spectrum, which was computed assuming a microturbulent velocity ranging from $10 \mathrm{~km} \mathrm{~s}^{-1}$ at the photosphere to $0.1 \times v_{\infty}$ in the outermost parts of the atmosphere. For each star, a subset of the model grid (encompassing the values of $T_{\text {eff }}$ and $\log g$ estimated from the spectral type) was convolved with the appropriate $V \sin i$ and $v_{\text {mac }}$ as described above. The resulting synthetic spectra, correctly shifted in RVs, were subsequently compared to the disentangled spectrum of each target. The quality of the fit was quantified by means of a $\chi^{2}$ analysis renormalized to 1.0 for the best-fit combination of $T_{\text {eff }}$ and $\log g$. The output of such a process is illustrated in Fig. 2 in the case of the primary component of Y Cyg. The uncertainties on $T_{\text {eff }}$ and $\log g$ were estimated from such figures. In practice, $T_{\text {eff }}$ and $\log g$ are correlated and giving a formal error on $T_{\text {eff }}$ and $\log g$ is not correct. However, we decided to take the width of the $\chi^{2}=2.0$ contour as a representative error. These values are reported in Table 3.

Surface abundances of $\mathrm{C}, \mathrm{N}$, and $\mathrm{O}$ were determined as in Martins et al. (2015): for a given set of $T_{\text {eff }}$ and $\log g$ models with different $\mathrm{C}, \mathrm{N}$, and $\mathrm{O}$ content were computed and subsequently compared to disentangled spectra. The goodness of fit was quantified by means of a $\chi^{2}$ analysis. In practice, we relied on the following lines:

- For carbon: C III 4068-70, C III 4153, C III 4156, C III 4163, C III 4187, C II 4267, C II 6578, and C II 6583.

- For nitrogen: N II 3995, N II 4004, N II 4035, N II 4041, N III 4044, N II 4236, N II 4447, N III 4511, N III 4515, N III 4518, N III 4524, N II 4530, N II 4601, N II 4607, N II 4614, N II 4621, N II 4630, N II 4788, N II 4803, N II 5001, N II 5005, and N II 5680.

- For oxygen: O II 3913, O II 3955, O II 4120, O II 4284, O II 4305, OII 4318, O II 4321, O II 4368, O II 4592, OII 4597, O II 4603, O II 4611, O II 4663, O II 4678, O II 4700, O II 4707, and O III 5592. 


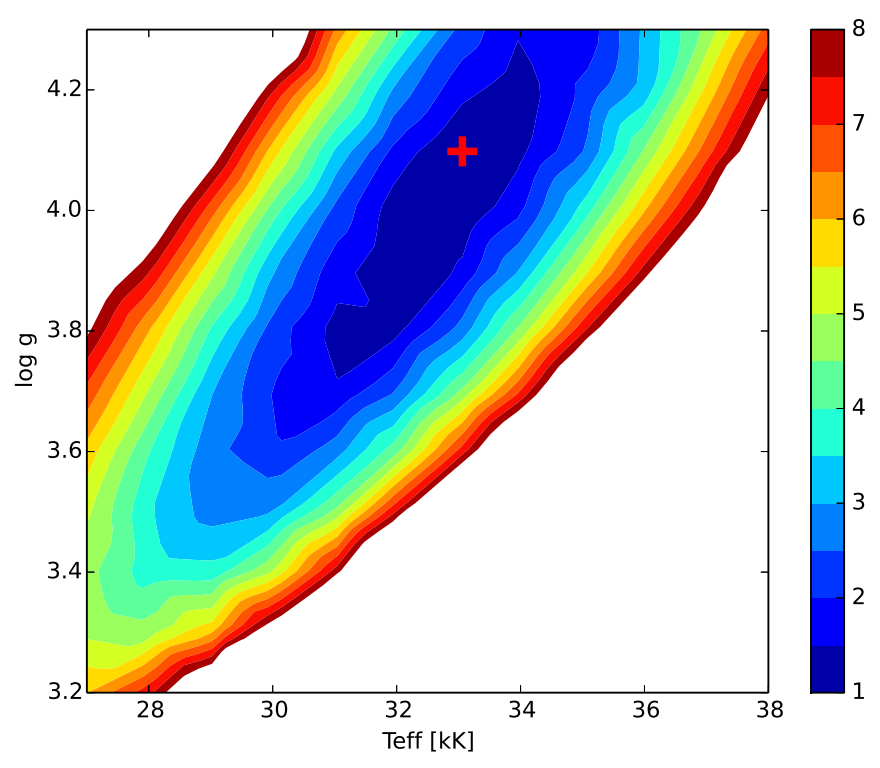

Fig. 2. Determination of $T_{\text {eff }}$ and $\log g$. Colors illustrate the value of $\chi^{2}$ for the fit of a synthetic spectrum with a given $T_{\text {eff }}-\log g$ combination to the observed disentangled spectrum of the primary component of Y Cyg. The red cross indicates the position at which $\chi^{2}=1.0$.

The number of lines used for each star depends on the effective temperature and quality of the spectra. We adopted $\mathrm{He} / \mathrm{H}=0.1$ in our computations. No significant deviation from this value was observed in our fits (see Fig. 3).

The derived parameters are given in Table 3 . The final bestfit models are shown in Fig. 3. For each system, the best fit of the individual components were co-added taking into account the luminosity ratio and RVs determined at the date at which the comparison spectrum was obtained. In general, the quality of the fits is good. Notable problems are encountered around Balmer lines, especially $\mathrm{H} \delta$, illustrating the difficulty to correctly disentangle broad lines that are never completely separated observationally. This is even worse when their wings contain lines from other elements.

\subsection{Photometry}

Except for DHCep, the five other systems display eclipses. We thus used the PHysics Of Eclipsing BinariEs (PHOEBE; Prša \& Zwitter 2005, v0.31a) software to model the HIPPARCos light curves (Perryman et al. 1997). For their analysis, we kept the parameters of the orbit fixed at their values obtained through the spectroscopic analysis (Sect. 3.1). The PHOEBE software enables modeling of the light curve and the RV curve of an object at the same time. This software is based on the Wilson \& Devinney code (Wilson \& Devinney 1971) and uses the Nelder \& Mead simplex fitting method to adjust all the input parameters to find the best fit to the light curve. In search of the best-fit model, we included the reflection effect in the computation of the light curve. The latter is reported as the brightening of one star due to irradiation by its companion and has a non-negligible impact on very luminous systems when the two components orbit very close to each other. The reflection effect can affect the curvature of the light curve in zones outside the eclipse. In the PHOEBE code reflection is treated according to the theory of Wilson (1990). We also fixed the effective temperatures to the values obtained in the previous section. The photometric parameters derived by PHOEBE are given in Table 4. The error bars are computed by fixing one parameter at a time and we allow the others to vary to reach the minimum of the $\chi^{2}$ corresponding to a $68.3 \%$ confidence level $(1 \sigma)$.

\section{Discussion}

\subsection{Comparison to previous analysis}

In this section we compare our results with those available in the literature. Table 5 gathers the published values of masses and radii for the systems we analyzed.

V478 Cyg was studied by Popper \& Hill (1991). Within the error bars, their results are in excellent agreement with our findings with the exception of the radius of the secondary, which is slightly larger in our study.

Popper \& Hill (1991) also analyzed V382 Cyg. Our radii are marginally consistent with their results, while our mass estimates are lower. The masses of Burkholder et al. (1997) are in better agreement with our findings. Burkholder et al. (1997) concluded that V382 Cyg was an interacting system since both stars fill their Roche lobe. Similar conclusions were reached by Harries et al. (1997) who obtained masses and radii in excellent agreement with our results.

The mass and radius of the primary component of Y Cyg obtained by Burkholder et al. (1997), Harmanec et al. (2014), and Simon et al. (1994) are consistent with our values. On the contrary, we find a less massive and more compact secondary. This system is the only one for which the phase coverage of our observations does not sample the maximum separation (Fig. 1). This probably affects our results and explains the differences with previous studies. The effective temperature and surface gravity derived by Simon et al. (1994) $-T_{\text {eff }}=34500$ (34200) $\mathrm{K}$ and $\log g=4.16$ (4.18) for the primary (secondary) - are similar to our determinations within the error bars. The same conclusion applies to the determination of the inclination of the system, i.e., $i=86^{\circ} 37 \pm 0^{\circ} 12$ for Simon \& Sturm and $i=88^{\circ} 0 \pm 2.0$ for us.

The dynamical mass determination of Burkholder et al. (1997) for AH Cep is consistent with our results. Our masses are $\sim 25 \%$ lower and our radii about $20 \%$ smaller than those of Bell et al. (1986).

The inclination of DH Cep was estimated by Sturm \& Simon (1994), who found $i=47 \pm 1^{\circ}$. Adopting this value, we obtain masses of $38.4 \pm 2.5$ and $33.4 \pm 2.2 M_{\odot}$ for the primary and secondary. Within the error bars these values are in agreement with those of Burkholder et al. (1997) and Hilditch et al. (1996). Our effective temperatures are consistent with those of Sturm \& Simon (1994), i.e., $T_{\text {eff }}=44000$ (43000) K for the primary (secondary), but we find surface gravities larger by $\sim 0.3$ dex; Simon \& Sturm report surface gravitie $\log g=3.95$ (4.03) for the primary (secondary). This likely reflects the difficulty to disentangle correctly the individual profiles of Balmer lines that are the main gravity indicators.

Finally, XZ Cep was studied by Harries et al. (1997) who obtained masses lower by $\sim 3 M_{\odot}$ compared to our determinations. On the contrary their radii are slightly larger. These differences are probably due to the contact nature of the system (see below).

\subsection{Evolutionary status}

Figure 4 shows the classical HR diagram and the $\log g-T_{\text {eff }}$ diagram. All systems but XZ Cep have both of their components on the main sequence as defined by single-star evolutionary tracks. $\mathrm{XZ}$ Cep is the most evolved system of our sample. For almost all stars the same track can explain the position of stars in both 

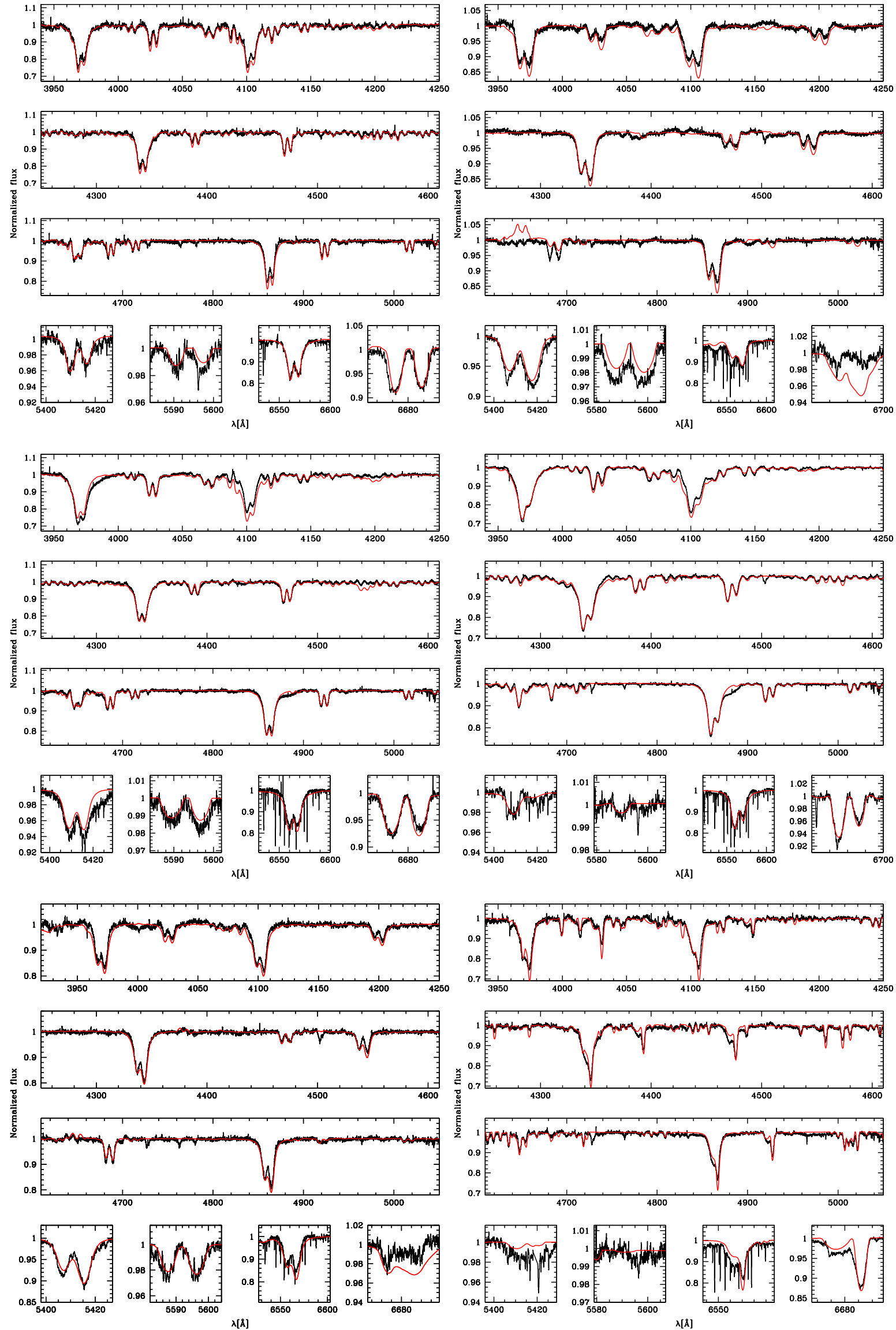

Fig. 3. Fit of one of the observed spectra of V478 Cyg (top left), V382 Cyg (top right), Y Cyg (middle left), AH Cep (middle right), DH Cep (bottom left), and XZ Cep (bottom right). The observed spectrum is shown in black and the best-fit model in red. The latter takes into account the luminosity ratio and RVs of both components. 
Table 3. Stellar parameters.

\begin{tabular}{lcccccccc}
\hline \hline Star & $\begin{array}{c}T_{\text {eff }} \\
{[\mathrm{kK}]}\end{array}$ & $\log g$ & $\log \frac{L}{L_{\odot}}$ & $\begin{array}{c}V \sin i \\
{\left[\mathrm{~km} \mathrm{~s}^{-1}\right]}\end{array}$ & $\begin{array}{c}v_{\mathrm{mac}} \\
{\left[\mathrm{km} \mathrm{s}^{-1}\right]}\end{array}$ & $\begin{array}{c}\mathrm{C} / \mathrm{H} \\
{\left[10^{-4}\right]}\end{array}$ & $\begin{array}{c}\mathrm{N} / \mathrm{H} \\
{\left[10^{-4}\right]}\end{array}$ & $\begin{array}{c}\mathrm{O} / \mathrm{H} \\
{\left[10^{-4}\right]}\end{array}$ \\
\hline DH Cep-1 & $44 \pm 3.0$ & $4.3 \pm 0.30$ & $5.40^{*}$ & 175 & 100 & $2.3_{-0.3}^{+0.4}$ & - & $15.0_{-4.0}^{+5.0}$ \\
DH Cep-2 & $41 \pm 2.0$ & $4.3 \pm 0.20$ & $5.50^{*}$ & 160 & 100 & $1.6_{-0.2}^{+0.2}$ & $1.0_{-1.0}^{+1.2}$ & $13.0_{-4.0}^{+1.5}$ \\
V382 Cyg-1 & $37 \pm 2.0$ & $3.8 \pm 0.15$ & $5.17 \pm 0.10$ & 260 & $0^{*}$ & $2.5_{-0.5}^{+0.5}$ & $0.7_{-0.7}^{+0.7}$ & $3.2_{-1.6}^{+1.6}$ \\
V382 Cyg-2 & $38 \pm 3.0$ & $3.8 \pm 0.20$ & $5.15 \pm 0.14$ & 240 & $0^{*}$ & $9.0_{-3.0}^{+3.6}$ & $1.5_{-0.4}^{+0.3}$ & $6.6_{-2.8}^{+3.0}$ \\
Y Cyg-1 & $33 \pm 1.5$ & $4.1 \pm 0.20$ & $4.57 \pm 0.08$ & 140 & $0^{*}$ & $1.5_{-0.4}^{+0.5}$ & $0.5_{-0.3}^{+0.3}$ & $3.7_{-1.0}^{+1.0}$ \\
Y Cyg-2 & $34 \pm 2.0$ & $4.2 \pm 0.20$ & $4.49 \pm 0.10$ & 160 & $0^{*}$ & $3.0_{-1.1}^{+2.0}$ & $0.8_{-0.4}^{+0.6}$ & $5.0_{-1.8}^{+3.0}$ \\
V478 Cyg-1 & $32 \pm 2.0$ & $3.9 \pm 0.20$ & $4.74 \pm 0.11$ & 120 & $0^{*}$ & $2.0_{-1.5}^{+0.6}$ & $0.8_{-0.3}^{+0.8}$ & $3.4_{-1.2}^{+1.5}$ \\
V478 Cyg-2 & $31 \pm 3.0$ & $3.7 \pm 0.30$ & $4.78 \pm 0.17$ & 120 & $0^{*}$ & $1.0_{-0.5}^{+0.5}$ & $0.35_{-0.35}^{+0.7}$ & $2.6_{-1.2}^{+1.6}$ \\
XZCep-1 & $28 \pm 1.0$ & $3.4 \pm 0.15$ & $5.05 \pm 0.06$ & 230 & $0^{*}$ & $<2.0$ & $0.6_{-0.6}^{+0.6}$ & $1.0_{-0.5}^{+1.7}$ \\
XZCep-2 & $24 \pm 3.0$ & $3.1 \pm 0.30$ & $4.79 \pm 0.22$ & 110 & $0^{*}$ & $0.3_{-0.2}^{+0.2}$ & $2.8_{-1.3}^{+3.4}$ & $0.5_{-0.5}^{+0.6}$ \\
AH Cep-1 & $31 \pm 3.0$ & $4.1 \pm 0.30$ & $4.41 \pm 0.17$ & 200 & $0^{*}$ & $1.0_{-0.9}^{+0.9}$ & $<0.2$ & $2.8_{-1.5}^{+1.2}$ \\
AH Cep-2 & $29 \pm 4.0$ & $4.2 \pm 0.30$ & $4.15 \pm 0.24$ & 170 & $0^{*}$ & $<2.0$ & $0.35_{-0.25}^{+0.35}$ & $2.3_{-0.9}^{+0.9}$ \\
\hline
\end{tabular}

Notes. A ${ }^{(*)}$ symbol indicated adopted values from Martins et al. (2005). Luminosities are computed from $T_{\text {eff }}$ and $R_{\text {mean }}$ (Table 4). Abundances are number ratios.
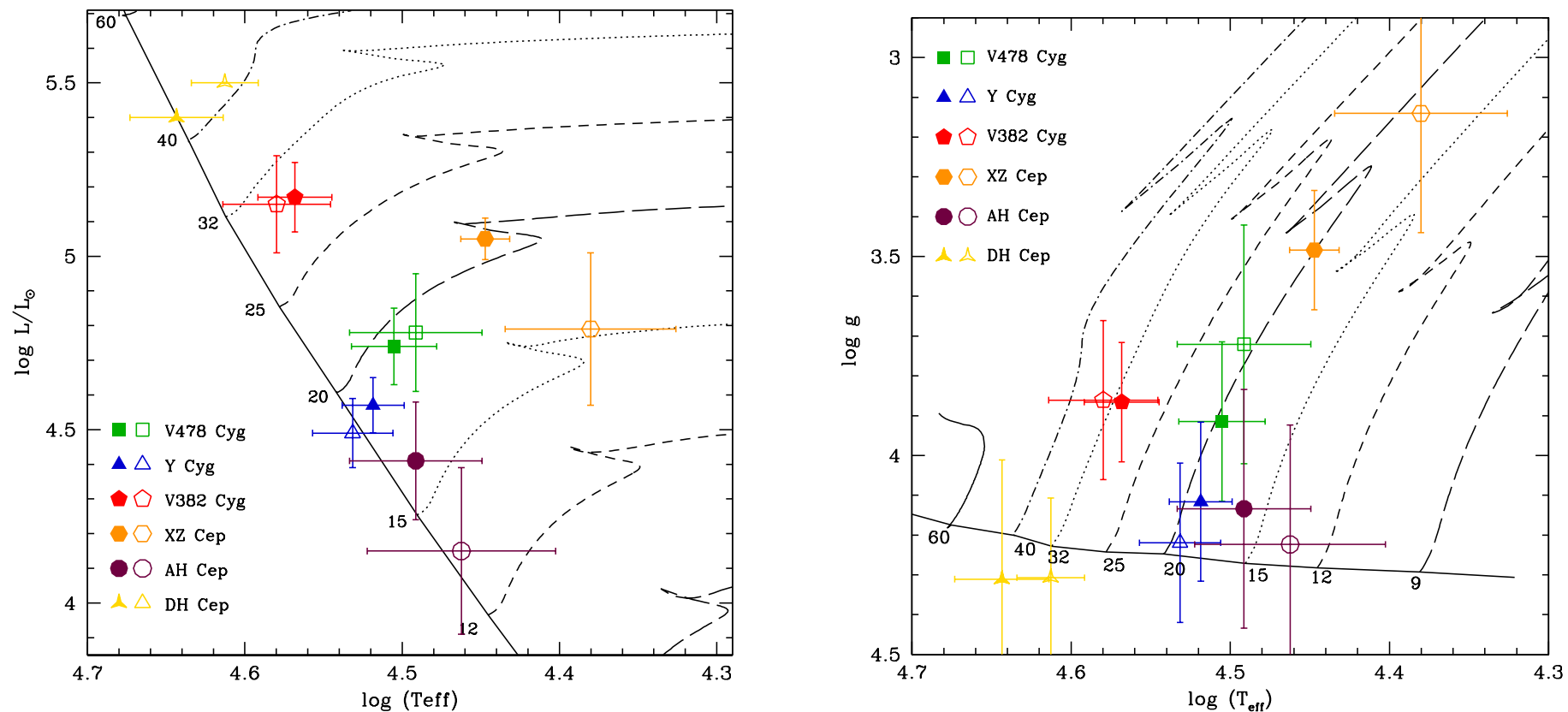

Fig. 4. Left: Hertzsprung-Russell diagram. Right: surface gravity as a function of effective temperature for the sample stars. Evolutionary tracks are from Ekström et al. (2012). Filled (open) symbols refer to the primary (secondary) of each binary system.

the HR and $\log g-T_{\text {eff }}$ diagrams. The secondary component of XZ Cep may be the only exception since its luminosity places it close to the $15 M_{\odot}$ track in the HR diagram while a mass of $20 M_{\odot}$ better reproduces its position in the $\log g-T_{\text {eff }}$ diagram. But we also note that within the error bars the 15 and $20 M_{\odot}$ tracks are relevant in both diagrams.

Table 6 provides an estimate of the Roche lobe size $\left(R_{\mathrm{L}}\right)$ for each component of the five eclipsing systems. This estimate was made using Eq. (2) of Eggleton (1983). In the third column of Table 6 we compare $R_{\mathrm{L}}$ to the stellar radius in the direction of the companion $\left(R_{\text {point }}\right)$ when possible, or to the mean radius when $R_{\text {point }}$ is not available (see Table 4). V478 Cyg, Y Cyg, and $\mathrm{AH}$ Cep are detached systems. The latter two have the largest ratios $R_{\mathrm{L}} / R_{\text {point }}$, which is consistent with their position very close to the ZAMS. V478 Cyg, which is more evolved, is on the verge of filling its Roche lobe (secondary star). V382 Cyg and XZ Cep are contact systems, in agreement with the findings of Harries et al. (1997). Given the above remarks regarding the position on the $\mathrm{HR}$ and $\log g-T_{\text {eff }}$ diagrams, XZ Cep has most likely experienced mass transfer and certainly follows an evolution different from that of single stars (see also Sect. 4.3). The properties of V382 Cyg in both evolutionary diagrams do not indicate deviation from single star evolution as strong as for XZ Cep: V382 Cyg 


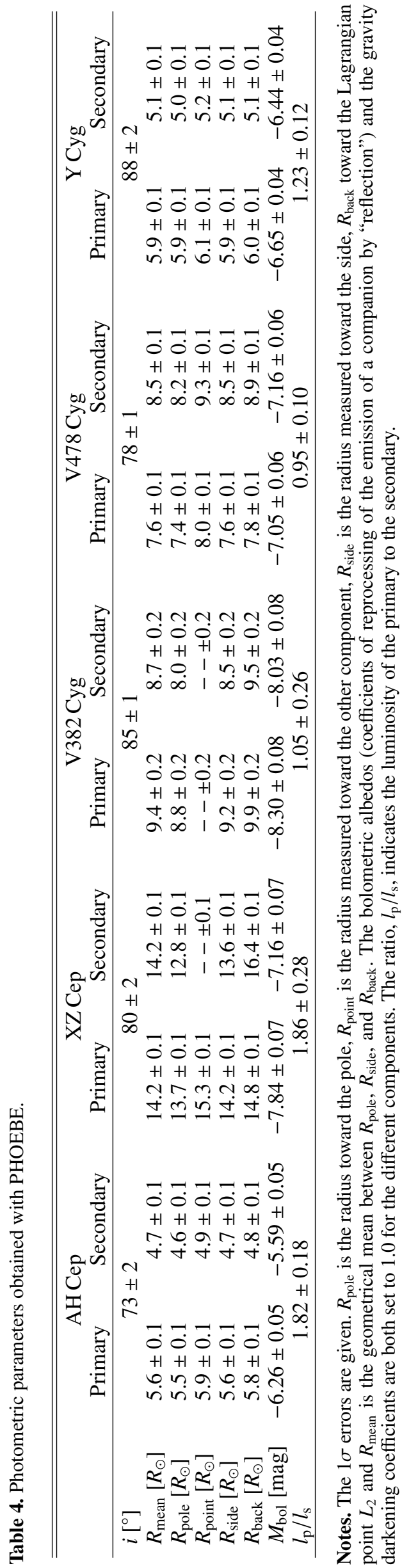

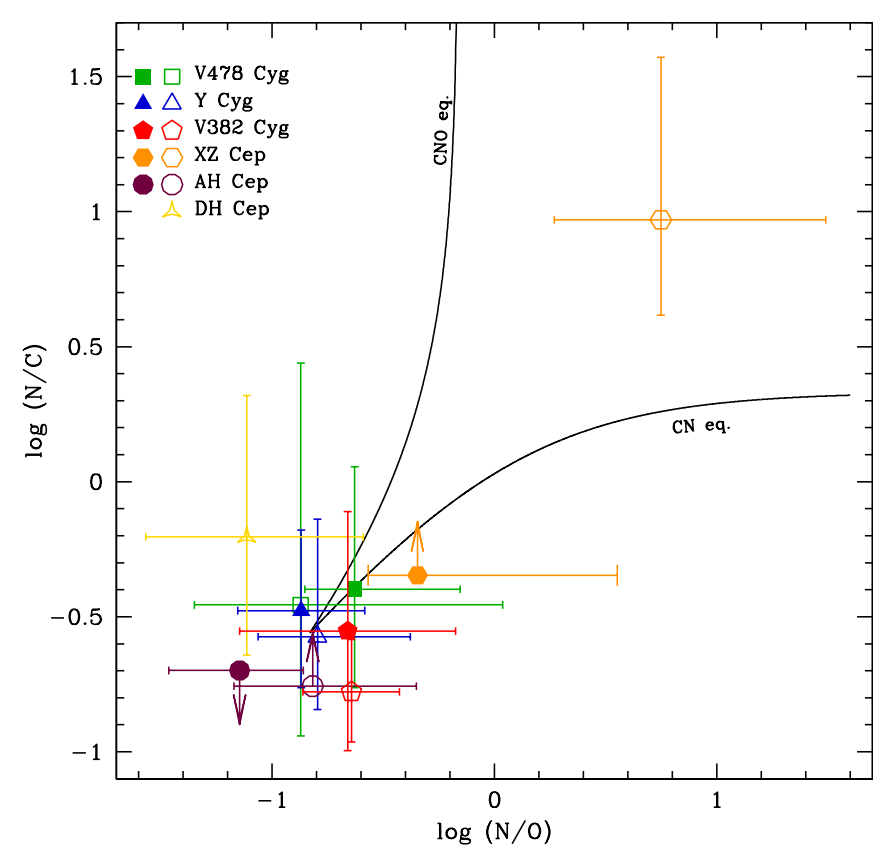

Fig. 5. Logarithm of N/C as a function of logarithm N/O. Solid lines indicate values for complete or partial $\mathrm{CN}(\mathrm{O})$ cycle. Symbols have the same meaning as in Fig. 4.

is thus probably only in an early contact phase so that deviation from single-star evolution has not appeared yet.

It is interesting to compare Y Cyg and V478 Cyg in light of Table 6: both stars have a mass ratio close to 1.0, dynamical masses around $15 M_{\odot}$, and similar orbital periods (just below three days). According to Fig. 4 Y Cyg is located closer to the ZAMS and is less evolved than V478 Cyg, which lies in the middle of the main sequence. This is reflected in Table 6 wherein the former system has its components filling a smaller fraction of its Roche lobe than the latter. We conclude that for an orbital period of about three days and stars with similar initial masses of about $15 M_{\odot}$ contact does not happen before the final portion of the main sequence.

\subsection{Surface abundances}

Figure 5 shows that most of the sample stars are barely evolved in terms of surface $\mathrm{CNO}$ abundances. Almost all stars have C, $\mathrm{N}$, and $\mathrm{O}$ abundance patterns consistent with no processing. The only exception is the secondary of $\mathrm{XZ} \mathrm{Cep,} \mathrm{which} \mathrm{is} \mathrm{signif-}$ icantly enriched. The ratios N/C and N/O are consistent with CNO burning.

Figure 6 shows a comparison of the sample stars with presumably single stars analyzed by Martins et al. (2015). We chose this study as a reference for comparison since it is based on data of similar quality and the method employed to determine the stellar parameters and surface abundances is the same as that used in the present study. The left panel of Fig. 6 shows the $\log g-T_{\text {eff }}$ diagram. The comparison stars are separated in two subsamples depending on their initial mass (above or below $\sim 28 M_{\odot}$ ) according to the tracks of Ekström et al. (2012). In the middle panel, the surface abundances of V478 Cyg, Y Cyg, XZ Cep, and AH Cep can be compared to the single stars with masses below $28 M_{\odot}$. V382 Cyg and DH Cep are compared to more massive single stars in the right panel. We see that in both mass ranges there is no difference between the binary and single 
Table 5. Mass and radius from the literature.

\begin{tabular}{|c|c|c|c|c|c|}
\hline Star & $\begin{array}{r}M_{\mathrm{p}} \\
{\left[M_{\odot}\right]}\end{array}$ & $\begin{array}{r}M_{\mathrm{s}} \\
{\left[M_{\odot}\right]}\end{array}$ & $\begin{array}{r}R_{\mathrm{p}} \\
{\left[R_{\odot}\right]}\end{array}$ & $\begin{array}{r}R_{\mathrm{S}} \\
{\left[R_{\odot}\right]}\end{array}$ & Reference \\
\hline \multirow[t]{2}{*}{ V478 Cyg } & $15.3 \pm 0.8$ & $14.6 \pm 0.8$ & $7.6 \pm 0.1$ & $8.5 \pm 0.1$ & this work \\
\hline & $16.6 \pm 0.9$ & $16.3 \pm 0.9$ & $7.43 \pm 0.12$ & $7.43 \pm 0.12$ & Popper \& Hill (1991) \\
\hline \multirow[t]{4}{*}{ Y Cyg } & $16.9 \pm 0.5$ & $16.0 \pm 0.5$ & $5.9 \pm 0.1$ & $5.1 \pm 0.1$ & this work \\
\hline & $16.8 \pm 0.5$ & $17.7 \pm 0.4$ & - & - & Burkholder et al. (1997) \\
\hline & $17.72 \pm 0.35$ & $17.73 \pm 0.30$ & $5.785 \pm 0.091$ & $5.816 \pm 0.063$ & Harmanec et al. (2014) \\
\hline & $17.57 \pm 0.27$ & $17.04 \pm 0.26$ & $5.93 \pm 0.07$ & $5.78 \pm 0.07$ & Simon et al. (1994) \\
\hline \multirow[t]{4}{*}{ V382 Cyg } & $26.1 \pm 0.4$ & $19.0 \pm 0.3$ & $9.4 \pm 0.2$ & $8.7 \pm 0.2$ & this work \\
\hline & $32.6 \pm 1.8$ & $22.9 \pm 1.3$ & $8.8 \pm 0.6$ & $7.4 \pm 0.6$ & Popper \& Hill (1991) \\
\hline & $29.7 \pm 1.1$ & $20.3 \pm 0.9$ & - & - & Burkholder et al. (1997) \\
\hline & $26.0 \pm 0.7$ & $19.3 \pm 0.4$ & $9.6 \pm 0.1$ & $8.4 \pm 0.1$ & Harries et al. (1997) \\
\hline \multirow[t]{2}{*}{ XZ Cep } & $18.7 \pm 1.3$ & $9.3 \pm 0.5$ & $14.2 \pm 0.1$ & $14.2 \pm 0.1$ & this work \\
\hline & $15.8 \pm 0.4$ & $6.4 \pm 0.3$ & $7.0 \pm 0.2$ & $140.5 \pm 0.2$ & Harries et al. (1997) \\
\hline \multirow[t]{3}{*}{ AH Cep } & $14.3 \pm 1.0$ & $12.6 \pm 0.9$ & $5.6 \pm 0.1$ & $4.7 \pm 0.1$ & this work \\
\hline & $16.2_{25}^{+6.0}$ & $13.3_{2.3}^{+5.5}$ & - & - & Burkholder et al. (1997) \\
\hline & $18.1 \pm 0.9$ & $15.9 \pm 0.8$ & $6.7 \pm 0.2$ & $6.2 \pm 0.2$ & Bell et al. (1986) \\
\hline \multirow[t]{3}{*}{ DH Cep ${ }^{1}$} & $38.4 \pm 2.5$ & $33.4 \pm 2.2$ & - & - & this work \\
\hline & $34.4_{-2.5}^{+2.8}$ & $29.8_{-2.4}^{+2.5}$ & - & - & Burkholder et al. (1997) \\
\hline & $32.7 \pm 1.7$ & $29.6 \pm 1.6$ & - & - & Hilditch et al. (1996) \\
\hline
\end{tabular}

Notes. 1- For DH Cep, we assume an inclination of $47^{\circ} \pm 1$, according to Sturm \& Simon (1994), to obtain the dynamical masses.

star samples: they cover the same area of the $\log (\mathrm{N} / \mathrm{C})-\log g$ diagram. As above, the only possible difference is the secondary star of XZ Cep. Although there is no direct comparison single stars close to this star, we see that its N/C ratio corresponds to predictions of single star models with initial masses above $25 M_{\odot}$ (and close to $40 M_{\odot}$ ), while its estimated mass is in the range 8-20 $M_{\odot}$ (see Sect. 4.4). It is thus likely that in this evolved system binarity has affected the surface chemical composition. For the other five systems, there is no evidence for such an effect, even for V382 Cyg in which both components (barely) fill their Roche lobe. In Fig. 6 we also separated comparison stars according to whether they have $V \sin i$ higher than $120 \mathrm{~km} \mathrm{~s}^{-1}$ or not. The high- $V \sin i$ sample encompasses the range of projected rotational velocities of the binary components. Here again, there is no clear distinction between binaries and single stars.

We thus conclude that in the binary sample we studied, detached systems do not show clear differences in their surface abundances compared to single stars. For interacting systems, large N/C may be encountered (XZ Cep) or not (V382 Cyg). A certain time may be required after the beginning of the mass transfer to see its effects on surface abundances.

Garland et al. (2017) studied a sample of 33 B-type binary systems in the LMC, all of which are located on the main sequence. These authors did not perform a disentangling of the individual spectra but determined the stellar parameters of the primaries with atmosphere models. They used various assumptions regarding contamination of their spectra by the secondaries to infer the impact on the derived parameters. They estimated nitrogen surface abundances and projected rotational velocities and concluded that primary stars of their binary systems do not show differences in terms of surface enrichment compared to B-type single stars. They argued that they may have evolved effectively as single stars with low rotational velocities.

Mahy et al. (2017) determined the stellar parameters and surface abundances of the O7.5If+O9I(f) system HD 166734. Their analysis indicates that this is a detached system and that the degree of chemical processing is not different from what is observed in single $\mathrm{O}$ supergiants.
Table 6. Size of the Roche lobe $\left(R_{\mathrm{L}}\right)$ and stellar radius toward the companion $\left(R_{\text {point }}\right)$, all in units of solar radius.

\begin{tabular}{lccc}
\hline \hline Star & $R_{\mathrm{L}}$ & $R_{\text {point }}$ & $R_{\mathrm{L}} / R_{\text {point }}$ \\
\hline V478 Cyg-1 & 10.1 & $8.0 \pm 0.1$ & $1.26 \pm 0.1$ \\
V478 Cyg-2 & 9.9 & $9.3 \pm 0.1$ & $1.06 \pm 0.1$ \\
Y Cyg-1 & 10.9 & $6.1 \pm 0.1$ & $1.78 \pm 0.1$ \\
Y Cyg-2 & 10.4 & $5.2 \pm 0.1$ & $2.00 \pm 0.1$ \\
V382 Cyg-1 & 9.3 & $9.4 \pm 0.2^{1}$ & $0.99 \pm 0.2$ \\
V382 Cyg-2 & 8.0 & $8.7 \pm 0.2^{1}$ & $0.92 \pm 0.2$ \\
XZ Cep-1 & 16.7 & $15.3 \pm 0.1$ & $1.09 \pm 0.1$ \\
XZ Cep-2 & 12.1 & $14.2 \pm 0.1^{1}$ & $0.85 \pm 0.1$ \\
AH Cep-1 & 7.2 & $5.9 \pm 0.1$ & $1.22 \pm 0.1$ \\
AH Cep-2 & 6.8 & $4.9 \pm 0.1$ & $1.39 \pm 0.1$ \\
\hline
\end{tabular}

Notes. ${ }^{(1)} R_{\text {mean }}$ is used instead of $R_{\text {point }}$ since the latter is not available.

Mahy et al. (2011) analyzed the properties of the two components of the massive binary LZ Cep. They found that the secondary is extremely nitrogen and helium rich, while being carbon and oxygen poor. Quantitatively, $\log (\mathrm{N} / \mathrm{C})=1.6$ which, together with a $\log g$ of 3.1, places the star in the upper right corner of Fig. 7 (the present-day dynamical mass of the secondary is $\sim 6 M_{\odot}$ ). On the other hand, the primary is barely chemically evolved. Mahy et al. (2011) concluded that the surface abundances could be explained by an inefficient mass transfer from the secondary (which was initially the primary) toward the primary. The dynamical masses obtained by Mahy et al. for LZ Cep ( $\sim 16$ and $\sim 6 M_{\odot}$ for the primary and secondary) are significantly lower than the evolutionary masses $\left(25.3\right.$ and $\left.18.0 M_{\odot}\right)$ determined using single star evolutionary tracks. This is another indication that mass transfer occurred in that system according to Mahy et al. (2011). In any case, the degree of chemical enrichment is higher than expected for single stars of masses 5 to $25 M_{\odot}$, and would be consistent with the enrichment of a $\sim 50 M_{\odot}$ star. 

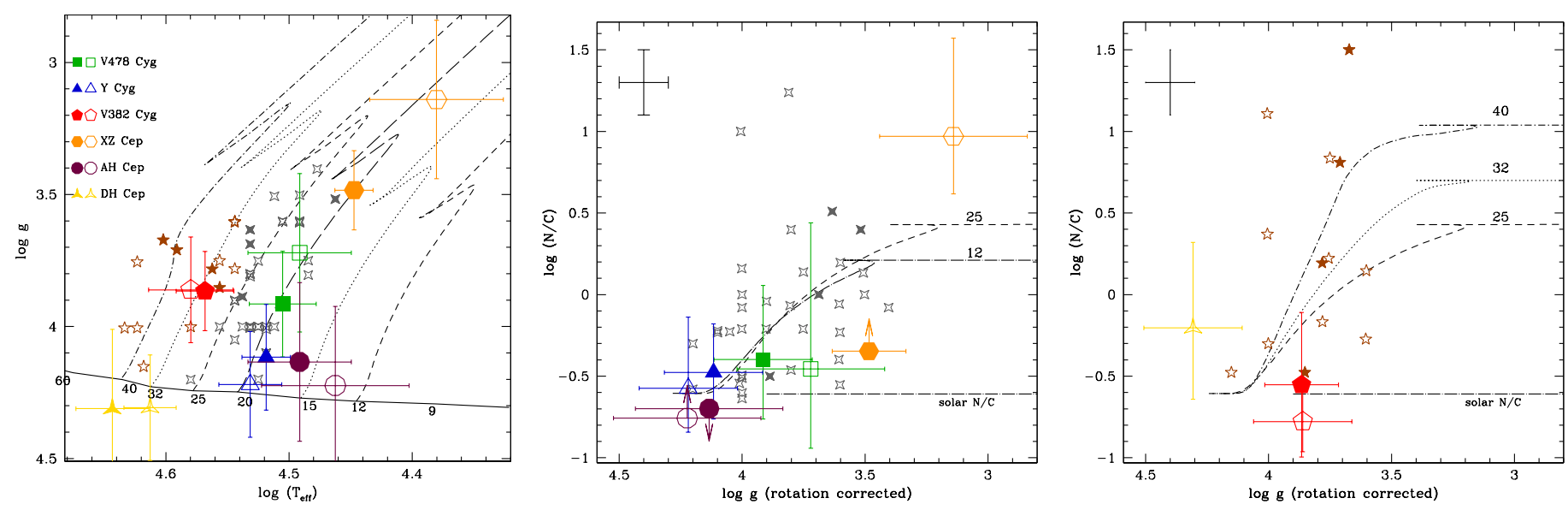

Fig. 6. Left: $\log g-T_{\text {eff }}$ (left) diagram for the sample stars together with comparison single stars from Martins et al. (2015). In the comparison sample, gray asterisks (brown stars) refer to stars less (more) massive than $\sim 28 M_{\odot}$. Filled symbols (stars and asterisks) refer to comparison stars with $V \sin i>120 \mathrm{~km} \mathrm{~s}^{-1}$. For clarity only a representative error bar for the comparison sample is shown. The evolutionary models including rotation of Ekström et al. (2012) are shown. Middle and right: $\log (\mathrm{N} / \mathrm{C})-\log g$ diagram for stars with masses lower than $28 M_{\odot}($ middle) and higher than $28 M_{\odot}(r i g h t)$. In all panels big symbols have the same meaning as in Fig. 4.

The surface abundances of the components of the system HD 149404 are similar to that of LZ Cep according to Raucq et al. (2016); see also Fig. 7. Since HD 149404 is not an eclipsing binary, there is no determination of its dynamical mass. The evolutionary masses reported by Raucq et al. (2016) are on the order $30 M_{\odot}$ assuming a standard initial rotation rate. As for LZ Cep, the secondary is thus highly enriched, as confirmed by its position in Fig. 7: the observed N/C ratio is larger by $\sim 0.8$ dex compared to the prediction of a $32 M_{\odot}$ model. Given these abundances and the asynchronous rotation of the system, Raucq et al. (2016) suggested that HD 149404 is in a post-Roche lobe overflow state. Such an interaction may have revealed internal layers of the secondary star, explaining the rather strong enrichment.

The massive evolved system LSS 3074 was studied by Raucq et al. (2017). The dynamical masses of the components are 14.8 and $17.2 M_{\odot}$ for the primary and secondary, respectively. These values (together with the estimated radii) are low for stars with spectral type O4f and O6-7(f). Raucq et al. determined lower limits of 0.94 (1.26) for the primary (secondary) $\log (\mathrm{N} / \mathrm{C})$ ratios. The primary is also helium rich $(\mathrm{He} / \mathrm{H}=0.25)$. Because the surface gravities of both components are close to 3.8, the stars are located in the upper part of Fig. 7. Raucq et al. (2017) indicate that LSS 3074 is likely an overcontact system that experienced Roche lobe overflow. Their simulations favor a relatively massive primary $\left(M=35 M_{\odot}\right)$. In that case, the N/C ratio determined for that star is high compared to predictions of evolutionary models (see Fig. 7). Compared to the two systems described above, it is worth noting that in the case of LSS 3074 both components show a high N/C ratio.

Both components of Plaskett's star (HD 47129) have initial masses above $40 M_{\odot}$ according to Linder et al. (2008). The primary has a high $\mathrm{N} / \mathrm{C}$ ratio, while the secondary is surprisingly chemically unevolved. The secondary has a high projected rotational velocity, which prompted Linder et al. to argue that this system experienced a recent mass transfer in which a small fraction of the mass removed from the primary was accreted by the secondary. This could explain the high rotation and absence of nitrogen enrichment and carbon depletion of the secondary. In Fig. 7 the primary is located in the upper part at a position consistent with high mass single stars. A strong magnetic field is present at the surface of the secondary Grunhut et al. (2013), which may have affected

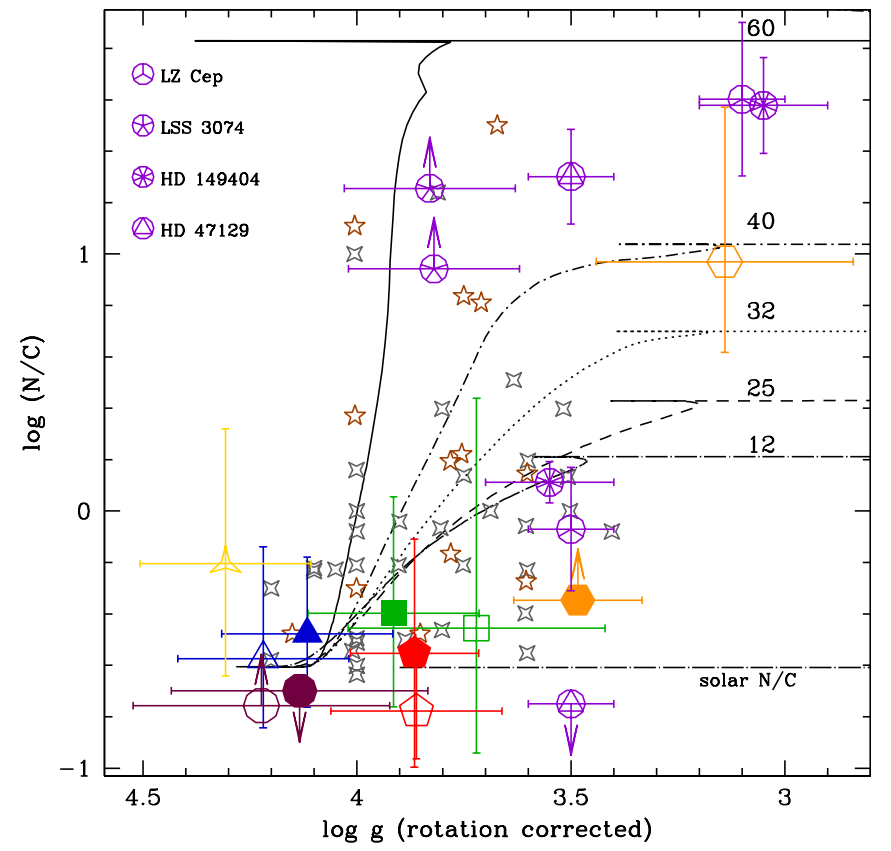

Fig. 7. Same as middle and right panels of Fig. 6 but with the evolved systems HD 47129 (Linder et al. 2008), LZ Cep (Mahy et al. 2011), HD 149404 (Raucq et al. 2016), and LSS 3074 (Raucq et al. 2017) added. Big symbols have the same meaning as in Fig. 4.

the interaction history and surface abundances of that system (e.g., Meynet et al. 2011).

From the above discussion, and keeping in mind that samples of binary stars for which surface abundances are available remain small, the following trend tends to emerge:

- Systems for which no evidence of mass transfer exist do not show a surface enrichment that is significantly different from single stars, at least within the uncertainties on the derived surface abundances. In other words, the additional role of tides on mixing in detached systems has a limited effect on surface abundances compared to mixing in single stars.

- On the contrary, in three systems that experienced mass transfer (HD 47129, LZ Cep, and HD 149404), the mass donor has a high N/C ratio, i.e., even higher than what is 

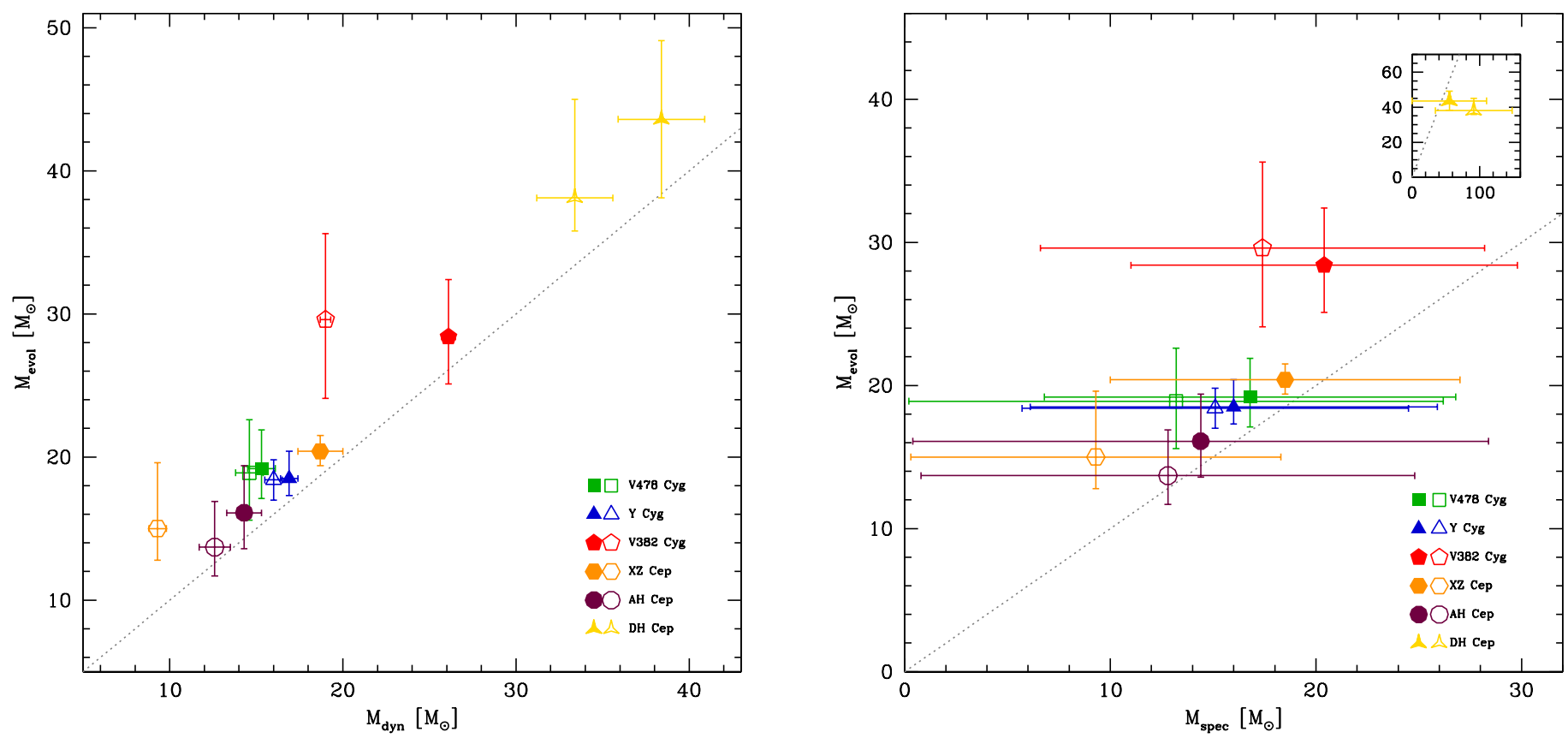

Fig. 8. Evolutionary mass as a function of dynamical mass (left) and spectroscopic mass (right). Symbols have the same meaning as in Fig. 4.

Table 7. Dynamical, evolutionary, and spectroscopic masses.

\begin{tabular}{lccc}
\hline \hline Star & $M_{\text {dyn }}$ & $M_{\text {evol }}$ & $M_{\text {spec }}$ \\
\hline V478 Cyg-1 & $15.3 \pm 0.8$ & $19.2_{-2.1}^{+2.7}$ & $16.8 \pm 10.0$ \\
V478 Cyg-2 & $14.6 \pm 0.8$ & $18.9_{-3.3}^{+3.7}$ & $13.2 \pm 13.0$ \\
Y Cyg-1 & $16.9 \pm 0.5$ & $18.5_{-1.2}^{+1.9}$ & $16.0 \pm 9.9$ \\
Y Cyg-2 & $16.0 \pm 0.5$ & $18.4_{-1.4}^{+1.4}$ & $15.1 \pm 9.4$ \\
V382 Cyg-1 & $26.1 \pm 0.4$ & $28.4_{-3.3}^{+4.0}$ & $20.4 \pm 9.4$ \\
V382 Cyg-2 & $19.0 \pm 0.3$ & $29.6_{-5.5}^{+6.0}$ & $17.4 \pm 10.8$ \\
XZCep-1 & $18.7 \pm 1.3$ & $20.4_{-1.0}^{+1.1}$ & $18.5 \pm 8.5$ \\
XZCep-2 & $9.3 \pm 0.5$ & $15.0_{-2.2}^{+4.6}$ & $9.3 \pm 9.0$ \\
AH Cep-1 & $14.3 \pm 1.0$ & $16.1_{-2.5}^{+3.3}$ & $14.4 \pm 14.0$ \\
AH Cep-2 & $12.6 \pm 0.9$ & $13.7_{-2.0}^{+3.2}$ & $12.8 \pm 12.0$ \\
DH Cep-1 & $38.4 \pm 2.5^{1}$ & $43.6_{-5.5}^{+5.5}$ & $55.2 \pm 55.0$ \\
DHCep-2 & $33.4 \pm 2.2^{1}$ & $38.1_{-2.3}^{+6.9}$ & $91.4 \pm 56.7$ \\
\hline
\end{tabular}

Notes. ${ }^{(1)}$ For DH Cep, the dynamical masses assume an inclination of $47^{\circ} \pm 1$, according to Sturm \& Simon (1994).

observed in single stars of the same initial mass and rotation rate for two systems (LZ Cep and HD 149404). Our results for XZ Cep follow the same trend: the secondary fills its Roche lobe and has a larger N/C than expected for its mass. In these systems mass transfers probably removed the external layers of the donor. This process exposes at the surface internal regions that are more chemically mixed, which is an effect similar to stellar winds for Wolf-Rayet stars. The mass gainer does not show peculiar surface abundances compared to single stars. This latter result is consistent with predictions of Langer et al. (2008), who showed that the accreted material is mixed and diluted in the pre-existing envelope. For HD 149404, both the primary and secondary show relatively high $\mathrm{N} / \mathrm{C}$ ratios, possibly indicating that the mass gainer was already chemically enriched (because of rotational mixing) before interaction. Finally, V382 Cyg just fills its Roche lobe but does not show signs of peculiar N/C.

Peculiar N/C ratios would thus be observed in the mass donor of post-mass transfer systems, possibly because of the removal of external layers and hence the appearance of internal layers deeply chemically processed at the surface. In absence of mass transfer, surface abundances of binary components would be indistinguishable from those of single stars. The case of the contact system V382 Cyg, for which no peculiar abundances are determined, suggests that the above picture is only partial or that the system was caught at the very beginning of the mass transfer episode when significant envelope removal did not occur yet. Obviously, this tentative picture needs to be confirmed by analysis of additional evolved systems. Such a task is difficult because the estimates of initial masses in interacting systems are based on assumptions on the efficiency of mass transfer, the initial orbital parameters, and the initial mass ratio (e.g., Wellstein et al. 2001; de Mink et al. 2009; Raucq et al. 2017). In addition, evolved systems are not spherically symmetric and the use of 1D atmosphere models may be questioned (Palate et al. 2013).

\subsection{Stellar masses}

In Table 7 we present three mass estimates: the dynamical mass $\left(M_{\text {dyn }}\right)$ results from the orbital solution; the evolutionary mass $\left(M_{\text {evol }}\right)$ estimated from the position in the HR diagram (Fig. 4, right panel); and the spectroscopic mass $\left(M_{\text {spec }}\right)$ obtained from the surface gravity and mean radius.

Figure 8 shows a comparison between these three masses. In the left panel, we see that $M_{\text {dyn }}$ and $M_{\text {evol }}$ are well correlated. However, there is a systematic upward shift indicating that evolutionary masses tend to be slightly larger (by a few solar masses) than dynamical masses. Quantitatively, the former are $16.5 \pm 10.6 \%$ higher than the latter with differences ranging between 8 and $38 \%$. 
Weidner \& Vink (2010) studied a large sample of binaries and concluded that there was no difference between dynamical and evolutionary masses. The only system in common with our study is V478 Cyg for which Weidner \& Vink (2010) give $M_{\text {dyn }}=16.6 \pm 9.0 M_{\odot}\left(16.3 \pm 9.0 M_{\odot}\right.$ for the secondary $)$ and $M_{\text {evol }}=18_{-5}^{+4} M_{\odot}$. The dynamical masses are in good agreement with our measurements. The evolutionary masses of Weidner \& Vink (2010) are slightly lower than ours, but consistent within the error bars. Vink \& Weidner relied on the tracks of Meynet \& Maeder (2003), which are less luminous for a given initial mass than those of Ekström et al. (2012) and those used here. With the 2003 Geneva tracks, one finds $M_{\text {evol }}=20.0_{-1.7}^{+2.3} M_{\odot}$ for the primary of V478 Cyg, i.e., in reasonable agreement with Weidner \& Vink (2010). The different conclusions reached by us and Vink \& Weidner regarding the discrepancy between evolutionary and dynamical masses is thus not clear. A dedicated analysis of their sample with our method is required to shed more light on this issue.

The right panel of Fig. 8 shows that the uncertainty on the spectroscopic masses are too large to allow any meaningful conclusion regarding the comparison between evolutionary and spectroscopic masses. This is partly due to the difficulty of the disentangling technique to correctly separate the Balmer lines of the components of the systems, and thus to the difficulty of determining surface gravities.

\subsection{Periods and synchronization}

Table 8 summarizes the rotational and orbital periods of each star/system. Because all systems but DH Cep are eclipsing and assuming that the rotational axis is parallel to the orbital axis, the rotational period can be obtained from $V \sin i$ and the radius can be obtained from the fit of the light curve. In two systems (Y Cyg and AH Cep) the rotational periods are shorter than the orbital one. Since tides tend to synchronize the rotation of stars with the orbital period (e.g., Zahn 1977), one can conclude that in these two systems the stars have not finished spinning down yet and that these systems were initially rotating with equatorial velocities larger than $150 \mathrm{~km} \mathrm{~s}^{-1}$. Interestingly, these systems are also the closest to the ZAMS, together with DH Cep for which no rotational period can be determined.

For V382 Cyg both periods are the same within the error bars, indicating that synchronization has been reached. This is also partly true for V478 Cyg.

XZ Cep is a peculiar case since the primary has $P_{\text {rotation }}<$ $P_{\text {orb }}$ while the opposite is true for the secondary. Given that this is the most evolved system of our sample and that the secondary fills its Roche lobe, this could indicate that mass and angular momentum transfer has lead to spinup of the mass gainer and spindown of the mass donor.

A better investigation of the effect of stellar evolution and mass transfer on rotational and orbital periods requires dedicated models. Such a study will be presented in a subsequent paper.

\section{Conclusion}

In this paper we have studied six Galactic massive binaries. We obtained high-resolution spectroscopy at different orbital phases. A spectral disentangling technique was applied to yield the individual spectra of each component. These spectra were subsequently analyzed with atmosphere models and synthetic spectra to provide the stellar parameters and $\mathrm{CNO}$ surface abundances. The results are:
Table 8. Rotational and orbital periods.

\begin{tabular}{lccc}
\hline \hline Star & $\begin{array}{c}P_{\text {rot }} \\
{[\mathrm{d}]}\end{array}$ & $\begin{array}{c}P_{\text {orb }} \\
{[\mathrm{d}]}\end{array}$ & $P_{\text {rotation }} / P_{\text {orb }}$ \\
\hline V478 Cyg-1 & $3.14 \pm 0.33$ & 2.88086 & $1.09 \pm 0.11$ \\
V478 Cyg-2 & $3.51 \pm 0.36$ & & $1.22 \pm 0.13$ \\
Y Cyg-1 & $2.13 \pm 0.19$ & 2.99633 & $0.71 \pm 0.06$ \\
Y Cyg-2 & $1.61 \pm 0.14$ & & $0.54 \pm 0.05$ \\
V382 Cyg-1 & $1.82 \pm 0.12$ & 1.88555 & $0.97 \pm 0.06$ \\
V382 Cyg-2 & $1.83 \pm 0.13$ & & $0.97 \pm 0.07$ \\
XZ Cep-1 & $3.08 \pm 0.19$ & 5.09725 & $0.60 \pm 0.02$ \\
XZ Cep-2 & $6.43 \pm 0.66$ & & $1.26 \pm 0.16$ \\
AH Cep-1 & $1.36 \pm 0.09$ & 1.77473 & $0.77 \pm 0.04$ \\
AH Cep-2 & $1.34 \pm 0.10$ & & $0.76 \pm 0.04$ \\
DH Cep-1 & $<2.52$ & 2.11095 & $<1.19$ \\
DH Cep-2 & $<3.54$ & & $<1.68$ \\
\hline \multicolumn{4}{c}{} \\
\hline
\end{tabular}

- Five of the six systems have their components located on the main sequence (as defined by single-star evolutionary tracks). For the sixth system, one component is evolved.

- Evolutionary masses determined using the single star evolutionary tracks of Ekström et al. (2012) are on average 16.5\% higher than dynamical masses.

- Three systems are detached (Y Cyg, V478 Cyg, and AH Cep), two have at least one star filling its Roche lobe (V382 Cyg and XZ Cep), and no information is available for DH Cep since it is not eclipsing.

- The CNO surface abundances of the three detached systems are little affected by stellar evolution and binarity. They are consistent with abundances of single stars within the uncertainties. Similar conclusions apply to the components of the contact system V382 Cyg and to the primary of XZ Cep. The $\mathrm{N} / \mathrm{C}$ ratio of the secondary of XZ Cep is higher than what is expected for single stars with similar initial masses (according to the evolutionary tracks of Ekström et al. 2012).

- Comparison of the derived surface abundances to those of systems known to have experienced mass transfer suggests the following trend: tides do not significantly affect the surface abundances of detached systems, while mass transfer, through the removal of external layers, can make chemically processed material appear at the surface. It may take some time after the onset of mass transfer before such effects are visible.

- Some systems have reached synchronization, while others may still be in a spin-down phase. In the contact binary XZ Cep mass transfer has probably affected the rotational periods.

The trends emerging from our results, especially regarding the effect of binarity on surface abundances, need to be confirmed by analysis of additional detached and post-mass transfer systems.

Acknowledgements. We thank an anonymous referee for a prompt and positive report. We thank the Observatoire de Haute Provence for assistance with the T-193 telescope and the SOPHIE instrument. We warmly thank John Hillier for making the code CMFGEN available to the community and for constant help with it. L.M. acknowledges support from the Fonds National de la Recherche Scientifique (F.R.S.-F.N.R.S.) and through the ARC grant for Concerted Research Actions, financed by the French Community of Belgium (WalloniaBrussels Federation).

\section{References}

Bell, S. A., Hilditch, R. W., \& Adamson, A. J. 1986, MNRAS, 223, 513 
F. Martins et al.: Properties of massive binaries

Bouchy, F., Hébrard, G., Udry, S., et al. 2009, A\&A, 505, 853

Bouchy, F., Díaz, R. F., Hébrard, G., et al. 2013, A\&A, 549, A49

Burkholder, V., Massey, P., \& Morrell, N. 1997, ApJ, 490, 328

Chiosi, C., \& Maeder, A. 1986, ARA\&A, 24, 329

Conti, P. S., Leep, E. M., \& Lorre, J. J. 1977, ApJ, 214, 759

de Mink, S. E., Cantiello, M., Langer, N., et al. 2009, A\&A, 497, 243

Eggleton, P. P. 1983, ApJ, 268, 368

Ekström, S., Georgy, C., Eggenberger, P., et al. 2012, A\&A, 537, A146

Garcia, M., Herrero, A., Najarro, F., Lennon, D. J., \& Alejandro Urbaneja, M. 2014, ApJ, 788, 64

Garland, R., Dufton, P. L., Evans, C. J., et al. 2017, A\&A, 603, A91

Gies, D. R. 2003, in A Massive Star Odyssey: From Main Sequence to Supernova, eds. K. van der Hucht, A. Herrero, \& C. Esteban, IAU Symp. 212,91

Gray, D. F. 1976, The observation and analysis of stellar photospheres (New York: Wiley-interscience), 484

Grevesse, N., Asplund, M., Sauval, A. J., \& Scott, P. 2010, Ap\&SS, 328, 179

Grin, N. J., Ramírez-Agudelo, O. H., de Koter, A., et al. 2017, A\&A, 600, A82

Grunhut, J. H., Wade, G. A., Leutenegger, M., et al. 2013, MNRAS, 428, 1686

Grunhut, J. H., Wade, G. A., Neiner, C., et al. 2017, MNRAS, 465, 2432

Hadrava, P. 1995, A\&AS, 114, 393

Harmanec, P., Holmgren, D. E., Wolf, M., et al. 2014, A\&A, 563, A120

Harries, T. J., Hilditch, R. W., \& Hill, G. 1997, MNRAS, 285, 277

Hilditch, R. W., Harries, T. J., \& Bell, S. A. 1996, A\&A, 314, 165

Hillier, D. J., \& Miller, D. L. 1998, ApJ, 496, 407

Huang, W., Gies, D. R., \& McSwain, M. V. 2010, ApJ, 722, 605

Hunter, I., Brott, I., Lennon, D. J., et al. 2008, ApJ, 676, L29

Kobulnicky, H. A., Kiminki, D. C., Lundquist, M. J., et al. 2014, ApJS, 213, 34

Langer, N., Cantiello, M., Yoon, S.-C., et al. 2008, in Massive Stars as Cosmic Engines, eds. F. Bresolin, P. A. Crowther, \& J. Puls, IAU Symp., 250, 167

Linder, N., Rauw, G., Martins, F., et al. 2008, A\&A, 489, 713

Maeder, A., \& Meynet, G. 2000, ARA\&A, 38, 143

Mahy, L., Martins, F., Machado, C., Donati, J.-F., \& Bouret, J.-C. 2011, A\&A 533, A9

Mahy, L., Rauw, G., De Becker, M., Eenens, P., \& Flores, C. A. 2013, A\&A, 550, A27

Mahy, L., Damerdji, Y., Gosset, E., et al. 2017, A\&A, in press, DOI: 10.1051/0004-6361/201730674
Mandel, I., \& de Mink, S. E. 2016, MNRAS, 458, 2634

Martins, F. 2011, Bulletin de la Societe Royale des Sciences de Liege, 80, 29

Martins, F., Schaerer, D., \& Hillier, D. J. 2005, A\&A, 436, 1049

Martins, F., Hervé, A., Bouret, J.-C., et al. 2015, A\&A, 575, A34

Mathis, S., \& Remus, F. 2013, in Lect. Notes Phys. 857, eds. J.-P. Rozelot, \& C. Neiner (Berlin Springer Verlag), 111

Meynet, G., \& Maeder, A. 2003, A\&A, 404, 975

Meynet, G., Eggenberger, P., \& Maeder, A. 2011, A\&A, 525, L11

Nieva, M.-F., \& Przybilla, N. 2014, A\&A, 566, A7

Palate, M., Rauw, G., Koenigsberger, G., \& Moreno, E. 2013, A\&A, 552, A39

Pavlovski, K., \& Hensberge, H. 2010, in Binaries - Key to Comprehension of the Universe, eds. A. Prša, \& M. Zejda, ASP Conf. Ser., 435, 207

Penny, L. R., \& Gies, D. R. 2009, ApJ, 700, 844

Perryman, M. A. C., Lindegren, L., Kovalevsky, J., et al. 1997, A\&A, 323, L49

Popper, D. M., \& Hill, G. 1991, AJ, 101, 600

Prša, A., \& Zwitter, T. 2005, ApJ, 628, 426

Ramírez-Agudelo, O. H., Simón-Díaz, S., Sana, H., et al. 2013, A\&A, 560, A29

Raucq, F., Rauw, G., Gosset, E., et al. 2016, A\&A, 588, A10

Raucq, F., Gosset, E., Rauw, G., et al. 2017, A\&A, 601, A133

Rauw, G., Sana, H., Gosset, E., et al. 2000, A\&A, 360, 1003

Sana, H., Gosset, E., \& Rauw, G. 2006, MNRAS, 371, 67

Sana, H., de Mink, S. E., de Koter, A., et al. 2012, Science, 337, 444

Sana, H., de Koter, A., de Mink, S. E., et al. 2013, A\&A, 550, A107

Sana, H., Le Bouquin, J.-B., Lacour, S., et al. 2014, ApJS, 215, 15

Simon, K. P., Sturm, E., \& Fiedler, A. 1994, A\&A, 292, 507

Simón-Díaz, S., \& Herrero, A. 2007, A\&A, 468, 1063

Sturm, E., \& Simon, K. P. 1994, A\&A, 282, 93

Ud-Doula, A., Owocki, S. P., \& Townsend, R. H. D. 2009, MNRAS, 392, 1022

Vink, J. S., de Koter, A., \& Lamers, H. J. G. L. M. 2001, A\&A, 369, 574

Weidner, C., \& Vink, J. S. 2010, A\&A, 524, A98

Wellstein, S., Langer, N., \& Braun, H. 2001, A\&A, 369, 939

Wilson, R. E. 1990, ApJ, 356, 613

Wilson, R. E., \& Devinney, E. J. 1971, ApJ, 166, 605

Wolfe, Jr., R. H., Horak, H. G., \& Storer, N. W. 1967, The machine computation of spectroscopic binary elements, eds. O. Struve, \& M. Hack, 251

Zahn, J.-P. 1977, A\&A, 57, 383

Zahn, J.-P. 1989, A\&A, 220, 112 